\title{
Update on the medical treatment of Graves' ophthalmopathy
}

This article was published in the following Dove Press journal:

International Journal of General Medicine

I December 2009

Number of times this article has been viewed

\section{Gregory J Griepentrog James A Garrity}

Department of Ophthalmology, Mayo Clinic, Rochester, MN, USA
Correspondence: James A Garrity Department of Ophthalmology, Mayo Clinic, 200 First Street, SW Rochester, MN 55905, USA

Tel + I 5072848538

Fax + I $50728446 \mid 2$

Email garrity.james@mayo.edu
Purpose: To review recent advances in the understanding of Graves' ophthalmopathy (GO) pathogenesis as well as discuss current and future medical management strategies.

Design: Interpretive essay.

Methods: Literature review and interpretation.

Results: Medical treatment of GO has slowly evolved during the past few decades and has been hampered by a poor understanding of the disease at a cellular and molecular level. Current treatment recommendations and guidelines therefore focus on nonspecific immuno-suppression. Newer classes of treatment agents hold promise to more selectively target underlying cellular and molecular alterations in GO.

Conclusion: Cooperation between individual patients, physicians and between differing medical centers, together with a refined understanding of the pathogenesis of GO, will lead to newer more-effective treatments for the disease and improve patient quality of life.

Keywords: Graves' ophthalmopathy, thyroid eye disease, thyroid-associated ophthalmopathy

\section{Introduction}

Graves' ophthalmopathy (GO) is the most common cause of orbital disease in adults and develops in $25 \%$ to $50 \%$ of patients with Graves' disease (GD). ${ }^{1}$ Although thyroid manifestations of GD can be effectively managed, the prevention and ultimately treatment of GO has remained challenging. Most patients can be managed with nonsurgical treatment, which will be the focus of this article. ${ }^{2}$ The surgical rehabilitation of GO has been reviewed by others. ${ }^{3,4}$ Here, we briefly review recent advances in the understanding of GO pathogenesis as well as discuss current and future medical management strategies.

\section{Disease pathogenesis}

Depending on the diagnostic criteria utilized GO can be seen in $25 \%$ to $50 \%$ of patients with GD. The annual incidence of GO is approximately 16 in 100,000 women and 3 in 100,000 men. ${ }^{5}$ The clinical course of GO differs from most autoimmune disorders in that there is typically an active phase with remission. ${ }^{6}$ Symptoms and signs of GO range from periorbital pain, conjunctival injection, chemosis, photophobia, diplopia, grittiness, lid lag and retraction, to proptosis, ophthalmoplegia, corneal ulceration, optic nerve dysfunction, and vision loss. Expansion of the orbital tissue (extraocular muscles, connective tissue, and orbital fat) within the confines of the rigid bony orbit, can explain the majority of these observed findings. 
A complex interplay between genetic and environmental factors contributes to susceptibility to GD. Although genetic predisposition to GD is well established in the literature, the precise role of genetic factors in the development of GO is unknown. ${ }^{7}$ Hereditary studies of GD have demonstrated a $30 \%$ to $40 \%$ concordance rate for monozygotic twins, while dizygotic twin studies reveal a lower concordance rate of approximately $5 \%{ }^{8,9}$ Non-twin siblings of patients with GD also have been noted to have an elevated risk when compared to the general population. ${ }^{8}$ Numerous case-controlled studies examining the role of genetic factors in the development of both GD and GO have been performed. Multiple loci for GO have been proposed including human leucocyte antigen (HLA, 6p21.3), cytotoxic T-lymphocyte antigen-4 (CTLA-4, 2q33), tumor necrosis factor $(T N F, 6 \mathrm{p} 21 \cdot 3)$, interferon- $\gamma(12 \mathrm{q} 14)$, intercellular adhesion molecule 1 (ICAM-1,19p13), and thyroid stimulating hormone receptor gene $(T S H-R, 14 \mathrm{q} 31){ }^{7}$ These results have not been confirmed or replicated in larger studies. It may be that both GD and GO are clinically heterogenous disorders making large-scale studies difficult. The possibility that GO may be predominantly influenced by environmental factors, rather than genetic predisposition, remains. ${ }^{6}$

A unified theory to explain the pathogenesis of GO does not exist. GO is often $(>90 \%)$, but not exclusively, associated with the onset of Graves' hyperthyroidism, thus suggesting a common autoimmune pathogenic mechanism. ${ }^{10}$ Once viewed as a bystander, orbital fibroblasts have been identified as a potential autoantibody target cell within the orbit. Thyroidstimulating hormone receptors (TSHr) have been identified in orbital fibroblasts of normal individuals and GO patients. ${ }^{1-13}$ At the mRNA level, patients with active GO have increased expression of TSHr versus patients with inactive disease. ${ }^{14}$ Furthermore, orbital fibroblasts show increased TSHr expression after adipocytic differentiation. ${ }^{15}$ Insulin-like growth factor-1 (IGF-1), another receptor on the orbital fibroblast cells, has also been shown to induce hyaluronan synthesis, ${ }^{16}$ while thyroid-specific antigens such as thyroid peroxidase and thyroglobulin have been detected in orbital tissues. ${ }^{17,18}$ Finally, autoantigens in extraocular muscles (calsequestrin) and orbital fibroblasts (collagen XIII) were reported to be specific and sensitive diagnostic markers of GO. ${ }^{19-21}$ It should be noted that although the autoantibody theory of GO pathogenesis is speculative as the evidence is thus far insufficient to link it fully to the clinical manifestations of GO. ${ }^{22}$

Once activated, sub-populations of orbital fibroblasts may release cytokines, produce glycosoaminoglycans (GAGs), and/or increase orbital fat through adipogenesis. ${ }^{23}$ Interleukin-6 fibroblast secretion promotes B-cell development and differentiation, while other chemokines recruit (IL-16, CXCL10, and IL-16) and activate (IL-8 and CXCL10) T-cells. ${ }^{23}$ Activated T cells trigger cytokine release (INF $\gamma, \mathrm{TNF} \alpha, \mathrm{PGD}_{2}$, and $15-\mathrm{dPGJ}_{2}$ ), thus potentiating further inflammation. ${ }^{23}$ One sub-population of activated orbital fibroblasts found predominantly in the orbital fat compartment termed "preadipocytes," displays the ability to differentiate into mature adipocytes. ${ }^{24} \mathrm{~A}$ separate orbital fibroblast sub-population found in connective tissues, that invests the extraocular muscles, may produce GAGs such as hyaluronic acid. Accumulation of these GAGs within the body of extraocular muscles leads to edema and muscle swelling. These changes may be observed radiographically (Figure 1). Volume measurement abnormalities of orbital adipose tissue and/or the extraocular muscles using computerized tomography, may be detected in $87 \%$ of Graves' patients with clinically detectable ophthalmopathy ${ }^{25}$ Conceptually the orbit has two compartments; a fat compartment and a muscle compartment. It has been noted that some patients with GO display adipose expansion as the primary manifestation of their disease, while others exhibit predominantly expansion of the muscle component. The predominance of one subpopulation of orbital fibroblasts within the orbit may help to explain this observation. ${ }^{24}$

Fibroblasts also have a number of tissue-specific phenotypes, which likely affects the selective involvement of the skin of the anterior lower legs, known as pretibial myxedema. Also termed Graves' dermopathy, this autoimmune extrathyroidal manifestation of Graves' disease is usually mild, and is seen in approximately $15 \%$ of patients with GO. Pretibial myxedema has been found to be a clinical marker of severe ophthalmopathy. ${ }^{26}$ Stimulation of local fibroblasts with a resulting production of significant amounts of GAGs, leads to the observed clinical manifestations. ${ }^{27}$

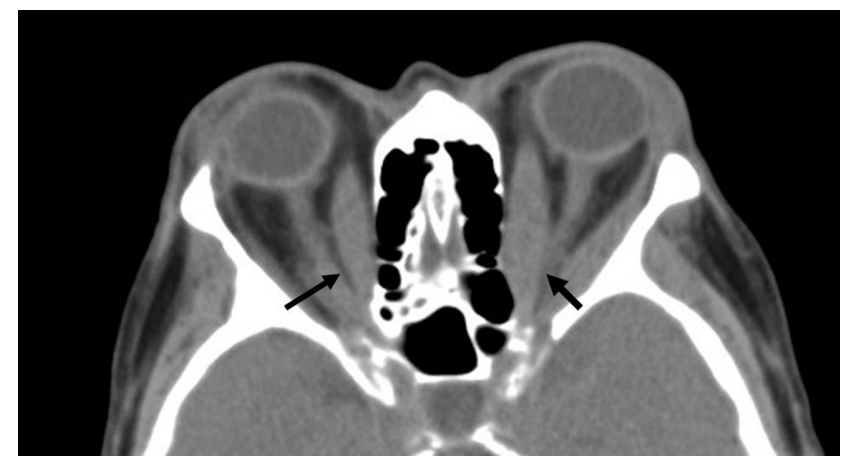

Figure I Axial CT scan of orbits, 67-year-old woman with Graves' ophthalmopathy with proptosis secondary to extraocular muscle expansion. Arrows demonstrate enlarged medial rectus muscles. Also note that left lateral rectus muscle is also enlarged. 


\section{Current medical treatment of Graves' ophthalmopathy}

The medical treatment of GO has gradually evolved during the past few decades. The relatively slow evolution of treatment may be explained partly by a poor understanding of $\mathrm{GO}$ at a cellular and molecular level, as well as challenges of disease classification at a clinical level. This has led to controversy and a wide variety of medical and surgical recommendations.

Graves' disease and Graves' ophthalmopathy are chronic disease processes with both physical and psychological manifestations. Education plays an important role to help with understanding patient concerns along with setting and aligning expectations. At times, patient concerns are very different than physician concerns. "Clinical success" in the treatment of GO has been a moving target. What is defined as "success" in the literature may in fact have little bearing on patients' perception of improvement. Current tools to evaluate quality of life (QOL) unfortunately lack questions on multiple issues that are important to most patients. ${ }^{28} \mathrm{An}$ updated QOL survey will be a useful outcome measurement tool in future clinical trials.

\section{Clinical grading}

There is no gold standard for grading the severity or activity of GO. This has led to difficulty comparing treatments for GO due to the wide variety of models used in clinical studies. Examples of severity grading systems include the modified NOSPECS (No symptoms or signs, Only signs, Soft tissue symptoms and signs, Proptosis, Extraocular muscle involvement, and Sight loss due to optic neuropathy) and the American Thyroid Association (ATA) grading systems. The American Thyroid Association (ATA) grading system divides patients according to clinical features and severity. Class I solely involve lid retraction or stare. Patients in Class II have soft tissue symptoms and signs (chemosis/edema), while patients in Class III have proptosis. Decreased motility and/or diplopia are characteristic of patients in Class IV. Finally, corneal exposure symptoms/signs or optic neuropathy are found in patients with Class V and Class VI disease, respectively. Used to assess disease activity, the Clinical Activity Score (CAS) is comprised of local manifestations of inflammation..$^{29}$ They include spontaneous retrobulbar pain, pain with eye movements, redness of the eyelids, redness of the conjunctiva, swelling of the eyelids, swelling of the caruncle and/or plica, and conjunctival edema. A score of 3 or greater is considered active GO. The VISA classification system combines aspects of both the CAS and NOSPECS systems. Core components include Vision (presence of absence of optic neuropathy), Inflammation ( $0-8$ or mild, moderate, or severe), Strabismus/motility (0-3), and Appearance/exposure (mild, moderate, or severe). ${ }^{30}$

The recent release of The Consensus Statement of the European Group on Graves' Orbitopathy (EUGOGO) has added refined recommendations to the management of GO. ${ }^{31}$ Although the authors do not advocate a detailed clinical grading or scoring system, they do recommend an initial segregation of patients into "mild," "moderate to severe," or "sight-threatening" categories, as well as an attempt to determine disease activity (Table 1).

\section{Initial management}

The initial management of patients with GO flows from widely accepted guidelines. These include the correction of thyroid dysfunction, smoking cessation counseling, and local symptomatic treatments for corneal exposure (lubricants and ointments), diplopia (prisms), and photophobia (dark lenses). Patients with uncontrolled hyperthyroidism are more likely to have severe GO than euthyroid patients. ${ }^{31-33}$ Although treatment modalities (thyroidectomy, antithyroid medications) of hyperthyroidism have similar outcomes in regards to $\mathrm{GO}$, treatment with radioiodine has been associated with progression in some patients with GO or newly diagnosed eye disease $(\sim 15 \%)$. This risk may be mitigated with a short course (3 months) of oral glucocorticoids (GCs), ${ }^{34}$ along with avoidance of post-operative hypothyroidism. ${ }^{35}$ Patients with risk factors such as smoking along with greater than mild GO are most likely to benefit from pretreatment. It has been suggested by some that only patients with preexisting moderate to severe GO should be pretreated with GCs.

Table I Severity classifications in Graves' ophthalmopathy (GO) ${ }^{31}$

I. Sight-threatening GO: patients with dysthyroid optic neuropathy (DON) and/or corneal breakdown. This category warrants immediate intervention.

2. Moderate to severe GO: patients without sight-threatening GO whose eye disease has sufficient impact on daily life to justify the risks of immunosuppression (if active) or surgical intervention (if inactive). Patients with moderate to severe GO usually have any one or more of the following: lid retraction $>2 \mathrm{~mm}$, moderate or severe soft tissue involvement, exophthalmos $>3 \mathrm{~mm}$ above normal for race and gender, inconstant or constant diplopia.

3. Mild GO: patients whose features of GO have only a minor impact on daily life insufficient to justify immunosuppressive or surgical treatment. They usually only have one or more of the following: minor lid retraction $(<2 \mathrm{~mm})$, mild soft tissue involvement, exophthalmos $<3 \mathrm{~mm}$ above normal for race and gender, transient or no diplopia, corneal exposure responsive to lubricants. 
There is a strong association between smoking and GO. Patients who smoke are more likely to have more severe $\mathrm{GO},{ }^{36,37}$ as well as an increased likelihood of GO progression after radioiodine therapy for hyperthyroidism. ${ }^{35}$ In addition, there appears to be a dose-related response between the number of cigarettes smoked per day and the probability of developing GO. ${ }^{38}$ Therefore, counseling to promote smoking cessation should take a central role in the management of all patients with GD. The precise mechanism underlying this association remains obscure, but free radical formation has been shown to stimulate orbital fibroblast proliferation. ${ }^{39}$

\section{Glucocorticoids}

Used for their immunosuppressive and antiinflammatory actions, glucocorticoids (GCs) are often utilized in the treatment of active GO. ${ }^{31}$ Since the early 1950 s, GCs have been administered through oral, local (retrobulbar or subconjunctival) or intravenous routes. Retrobulbar or subconjunctival GC injections have been found to be less effective than oral GCs. ${ }^{40}$ However, this route of administration is reasonable when oral or intravenous GCs are contraindicated.

Oral glucocorticoids have been a mainstay in the management of GO for many years. Recent randomized clinical trials have compared the efficacy of oral versus intravenous glucocorticoids (IVGCs). A recent prospective, single-blind, randomized study of 70 patients, directly compared oral GCs (initial dose $0.1 \mathrm{~g} /$ day tapering $0.01 \mathrm{~g} /$ week) versus IVGCs $(0.5 \mathrm{~g}$, then $0.25 \mathrm{~g}, 6$ weeks each) for the treatment of euthyroid patients with untreated, active, severe GO. ${ }^{43}$ The authors were able to use a lower cumulative dose of IVGCs (4.5 g) than previous studies, and they reported a treatment response rate (77 vs 51\%) favoring IVGCs.

A major concern of high-dose GC treatment is the risk of adverse side effects. Although GCs effectively suppress inflammation, they may be associated with a number of potential side effects including immunosuppression, hyperglycemia, osteoporosis and necrosis, together with weight gain. Cushing's syndrome is an almost unavoidable side effect of long-term use of oral glucocorticoids. The use of pulse IVGCs and large cumulative doses may result in serious liver toxicity and even death. Reporting of fatal acute liver failure led some authors to recommend a cumulative dose of no more than 6 g. ${ }^{42}$ The European consortium recommends pulsed IVGCs as the treatment of choice for patients with moderate to severe and active GO. ${ }^{31}$ In an accompanying editorial, concern was raised that for some patients with "moderate" disease and active GO, the risk-benefit ratio for IVCG may be weighted toward the unacceptable risk side.
The statement was given within the context that many patients will spontaneously improve, albeit not as quickly in the untreated group, given the natural history of the disease. ${ }^{44}$ Monitoring of liver function, along with other potential sideeffects such as hyperglycemia and increased blood pressure, during and after treatment is mandatory.

\section{Orbital radiation}

Orbital radiation (OR) has been used in the treatment of GO for more than 90 years. ${ }^{45}$ The rationale for the use of orbital radiotherapy stems from its anti-inflammatory effects and the radiosensitivity of activated orbital T-cells and fibroblasts. A common cumulative dose of radiation is 20 Gy per eye, fractionated over a 2-week period. Known complications of orbital radiation are fortunately rare but may include radiation retinopathy and cataract, ${ }^{46}$ There are also theoretic concerns of cancer induction which is estimated at $0.39 \% .{ }^{47}$

Orbital radiation was initially widely adopted, despite a lack of published data from controlled clinical trials clearly demonstrating its efficacy. To address this, several retrospective controlled clinical trials have been undertaken in the past decade. A controversial study by Gorman et al found that treatment with orbital radiation was no better than the natural history of the disease which is associated with spontaneous improvement. ${ }^{48}$ A recent review by Bradley et al evaluated the role of orbital radiation for GO by identifying 5 observational studies and 9 randomized controlled trials (RCTs) sufficient to meet inclusion criteria. ${ }^{49}$ The RCTs were qualitatively heterogeneous and only three of the 9 randomized trials were sham-controlled. ${ }^{48,50,51}$ The majority of the reviewed RCTs enrolled patients with moderately severe GO, although inclusion criteria ranged from one trial that enrolled patients solely with mild GO to another that enrolled patients with moderate to severe ophthalmopathy, including patients with optic neuropathy. The measured outcomes were similarly heterogenous and included changes in one or more clinical parameters such as lid fissure, proptosis, and extraocular muscle function, change in orbital computed tomography CT volume, change in NOSPECS class or grade, change in American Thyroid Association ophthalmopathy index, and change in major and minor criteria (Donaldson criteria). Two of the 3 sham-controlled trials demonstrated improved vertical range of motion in orbital radiation-treated subjects compared with controls.

There are a wide variety of recommendations for the use of orbital radiation in the treatment for GO. The European consortium recommends that, "Orbital irradiation (OR) should be considered in patients with active disease who have 
diplopia or restricted motility." ${ }^{31}$ Others recommend the use of orbital radiation as an adjunct to steroids, decompression, or both for recalcitrant disease. ${ }^{52} \mathrm{~A}$ larger, multicenter study may help to clarify some of the remaining issues surrounding the use of orbital radiation for GO.

\section{Future approaches to therapy}

A refined understanding of the pathogenesis of GO has led to the advent of new potential treatment targets (Figure 2). ${ }^{53,54}$ In the future, it may be possible to block receptor binding of TRAbs or IGF-1 autoantibodies with specific monoclonal anti-TSRr or anti-IGFr. ${ }^{10}$ Further downstream, orbital preadipocyte differentiation into mature adipocytes might also be inhibited. ${ }^{10}$

Inhibitors that block early steps in $\mathrm{T}$ and $\mathrm{B}$ cell activation have the potential to limit inflammatory cytokine and autoantibody production, respectively. Directed against CD20, which is expressed in pre-B cells and B cells, the monoclonal antibody rituximab (RTX) has been used in the management of chronic autoimmune diseases such as systemic lupus erythematosus, dermatomyositis, and rheumatoid arthritis (RA). ${ }^{55}$ RTX treatment induces necrosis and apoptosis of $\mathrm{CD} 20+$ cells. More recently, promising results have been reported using RTX in the treatment of GO. ${ }^{56,57}$ These initial studies were small but offer rationale for larger prospective, randomized, controlled trials. ${ }^{10}$ Other agents in this class include CTLA4-Ig (abatacept), a recombinant fusion protein

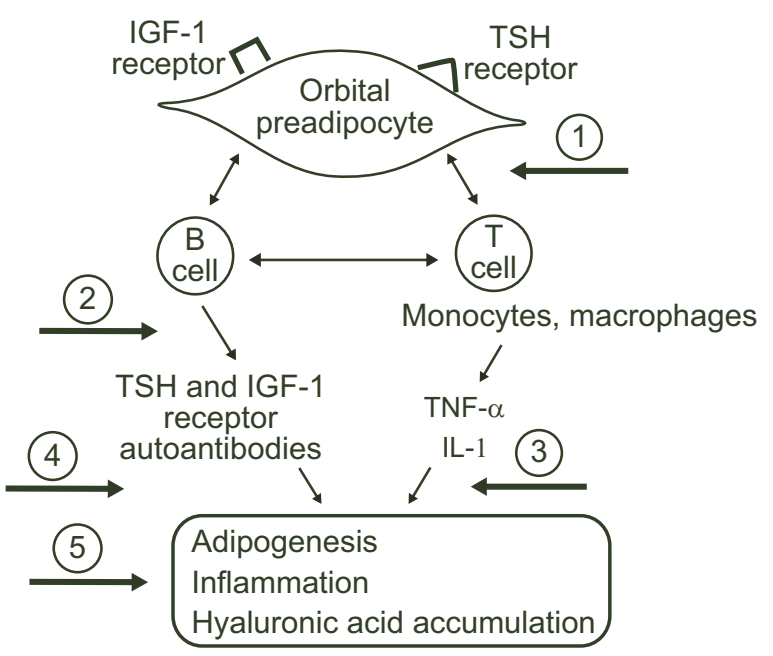

Figure 2 Proposed targets for agents of potential benefit in the treatment of Graves' ophthalmopathy. I) blocking T-cell co-stimulation, 2) depleting B cells, 3) inhibiting cytokine action, 4) targeting the insulin-growth factor I (IGF-I) receptor or the thyrotropin (TSH) receptor, and 5) preventing connective tissue remodeling. Adapted with permission from Garrity JA, Bahn RS. Pathogenesis of Graves Ophthalmopathy: Implications for Prediction, Prevention, and Treatment. Am J Ophthalmol. 2006; I42(I): 147-153. ${ }^{54}$ Copyright (C) 2008 Elsevier. that is a costimulatory or second signal-blocker of T- cell activation. Mimicking endogenous CTLA4, it competes with CD28 for B7.1 (CD80) and B7.2 (CD86) ligand binding, thus blocking a costimulatory signal required for optimal $\mathrm{T}$ cell activation. ${ }^{58,59}$ Abatacept was recently approved by the FDA for treatment of patients with RA, but to date, there are no published studies using the drug in patients with GO.

Anticytokine medications targeting tumor necrosis factor alpha (TNF- $\alpha$ ) or IL-1 have been approved for treatment of a number of auto-immune diseases. In particular, anti-TNF- $\alpha$ therapies with agents, such as etanercept and infliximab, have been of clinical value in difficult-to-treat RA. Studies using these agents to treat GO are limited. Etanercept is a TNF-receptor/immunoglobulin fusion molecule that has shown some promise in treating GO in a small pilot study. ${ }^{60}$ Infliximab, a monoclonal antibody to TNF- $\alpha$, was used to treat a patient with sight threatening GO. ${ }^{61}$ Anakinra, an IL-1 receptor antagonist is approved for the treatment of RA, but there are no reports of its use in GO.

\section{Conclusion}

A close relationship that emphasizes education and cooperation between patients and physicians is crucial to the management of GO. Medical therapy for GO has evolved little during the past few decades and generally focuses on conventional approaches of nonspecific immuno-suppression. This may be explained partly by a poor understanding of GO at a cellular and molecular level, as well as challenges of disease classification at a clinical level. On both fronts, progress is being made. Newer classes of treatment agents hold promise to more selectively target underlying cellular and molecular alterations in GO. Also, cooperation between multiple medical centers, as evidenced by the European Group on Graves' Orbitopathy (EUGOGO) and the recent formation of the International Thyroid Eye Disease Study Group (ITEDS), have the potential to better standardize the language and tests used in GO.

\section{Disclosures}

No financial support or grants were utilized for this study. The authors do not have financial disclosure or competing interests in the presented topic.

\section{References}

1. Bahn RS, Heufelder AE. Pathogenesis of Graves ophthalmopathy. N Engl J Med. 1993;329:1468-1475.

2. Bartley GB, Fatourechi V, Kadrmas EF, et al. The treatment of Graves' ophthalmopathy in an incidence cohort. Am J Ophthalmol. 1996;122:599-600. 
3. Goldberg RA. Advances in surgical rehabilitation in thyroid eye disease. Thyroid. 2008;18:989-995.

4. Schotthoefer EO, Wallace DK. Strabismus associated with thyroid eye disease. Curr Opin Ophthalmol. 2007;18:361-365.

5. Bartley GB, Fatourechi V, Kadrmas EF, et al. The incidence of Graves' ophthalmopathy in Olmsted County, Minnesota. Am J Ophthalmol. 1995;120:511-517.

6. Prabhakar BS, Bahn RS, Smith TJ. Current perspective on the pathogenesis of Graves' ophthalmopathy. Endocr Rev. 2003;24:802-835.

7. Bednarczuk T, Bamini G, Ploski R, Wall JR. Susceptibility genes in Graves' ophthalmopathy: searching for a needle in a haystack? Clin Endocrinol. 2007;67:3-19.

8. Brix TH, Kyvik KO, Hegedus L. What is the evidence for genetic factors in the etiology of Graves' disease? A brief review. Thyroid. 1998;8: $627-634$.

9. Brix TH, Chistensen K, Holm NV, et al. A populations-based study of Graves' disease in Danish twins. Clin Endocrinol. 1998;48:397-400.

10. Khoo TK, Bahn RS. Pathogenesis of Graves' ophthalmopathy: the role of autoantibodies. Thyroid. 17:1013-1018.

11. Feliciello A, Porcellini A, Ciullo I, Bonavolonta G, Avvediment EV, Fenzi G. Expression of thyrotropin-receptor mRNA in healthy and Graves' disease retro-orbital tissue. Lancet. 1993;342:337-338.

12. Heufelder AE, Dutton CM, Sakar G, Donovan KA, Bahn RS. Detection of TSH receptor RNA in cultured fibroblasts from patients with Graves' ophthalmopathy and pretibial dermopathy. Thyroid. 1993;3:297-300.

13. Starkey K, Janezic A, Jones G, Jordan N, Baker G, Ludgate M. Adipose thyrotrophin receptor expression is elevated in Graves' and thyroid eye diseases ex vivo and indicated adipogenesis in progress in vivo. $\mathrm{J} \mathrm{Mol}$ Endocrinol. 2003;30:369-380.

14. Bahn RS, Dutton CM, Natt N, Joba W, Spitzweg C, Heufelder AE. Thyrotropin receptor expression in Graves' orbital adipose/connective tissues: potential autoantigen in Graves' ophthalmopathy. $J$ Clin Endocrinol Metab. 1998;83:998-1002.

15. Wakelkamp IM, Bakker O, Baldeschi L, Wiersinga WM, Prummel MF. TSH-R expression and cytokine profile in orbital tissue of active vs inactive Graves' ophthalmopathy patients. Clin Endocrinol. 2003; $58: 280-287$

16. Smith TJ, Hoa N. Immunoglobulins from patients with Graves' disease induce hyaluronan synthesis in their orbital fibroblasts through the selfantigen, insulin-like growth factor-I receptor. J Clin Endocrinol Metab. 2004;89:5076-5080.

17. Lai OF, Zaiden N, Goh SS, et al. Detection of thyroid peroxidase mRNA and protein in orbital tissue. Eur J of Endocrinol. 2006;255:213-218.

18. Marino M, Lisi S, Pinchera A, et al. Glycosoaminoglycans provide a binding site for thyroglobulin in orbital tissues of patients with thyroidassociated ophthalmopathy. Thyroid. 2003;13:851-859.

19. Tani J, Wall JR. Autoimmunity against eye-muscle antigens may explain thyroid-associated ophthalmopathy. CMAJ. 2006;175:239.

20. Gopinath B, Musselman R, Beard N, et al. Antibodies targeting the calcium binding skeletal muscle protein calsequestrin are specific markers of ophthalmopathy and sensitive indicators of ocular myopathy in patients with Graves' disease. Clin Exp Immunol. 2006;145:56-62.

21. Gopinath B, Musselman R, Adams CL, Tani J, Beard N, Wall JR. Study of serum antibodies against three eye muscle antigens and the connective tissue antigen collagen XIII in patients with Graves' disease with and without ophthalmopathy - correlation with clinical features. Thyroid. 2006; 16:967-974.

22. Naik V, Khadavi N, Naik MN, et al. Biologic therapeutics in thyroidassociated ophthalmopathy: translating disease mechanism into therapy. Thyroid. 2008;18:967-971.

23. Kuriyan AE, Phipps RP, Feldon SE. The eye and thyroid disease. Curr Opin Ophthalmol. 2008;19:499-506.

24. Smith TH, Koumas L, Gagnon A, et al. Orbital fibroblast heterogeneity may determine the clinical presentation of thyroid-associated ophthalmopathy. J Clin Endocrinol Metab. 2002;87:385-392.
25. Forbes G, Gorman CA, Brennan MD, Gehring DG, Ilstrup DM, Earnest FT. Ophthalmopathy of Graves' disease: computerized volume measurements of the orbital fat and muscle. AJNR Am J Neuroradiol. 1986;7:651-656.

26. Fatourechi V, Bartley BG, Eghbali-Fatourechi GZ, Powell CC, Ahmed DD, Garrity JA. Graves dermopathy and acropachy are markers of severe Graves ophthalmopathy. Thyroid. 2003;13:1141-1144.

27. Khalilzadeh O, Mojazi Amiri H, Tahvildari M, et al. Pretibial myxedema is associated with polymorphism in exon 1 of CTLA-4 gene in patients with Graves' ophthalmopathy. Arch Dermatol Res. 2009;301: 719-723.

28. Bradley EA, Sloan JA, Novotny PJ, Garrity JA, Woog JJ, West SK. Evaluation of the National Eye Institute Visual Function Questionnaire in Graves' Opthalmopathy. Ophthalmology. 2006;113: $1450-1154$.

29. Mouritis MP, Prummel MF, Wiersinga WM, Koorneef L. Clinical activity score as a guide in the management of patients with Graves' ophthalmopathy. Clin Endocrinol (Oxf). 1997;47:9-14.

30. Doleman PJ, Rootman J. VISA classification for Graves' orbitopathy. Ophthal Plast Reconstr Surg. 2006;22:319-324.

31. Bartalena L, Baldeschi L, Dickinson AJ, et al. Consensus Statement of the European Group on Graves' Orbitopathy (EUGOGO) on Management of Graves' Orbitopathy. Thyroid. 2008;18:333-346.

32. Prummel MF, Wiersinga WM, Mouritis MP, Koornneef L, Berghout A, van der Gaag R. Amelioration of eye changes of Graves' ophthalmopathy by achieving euthyroidism. Acta Endocrinol (Copenh). 1989;21(Suppl 2):185-189.

33. Prummel MF, Wiersinga WM, Mouritis MP, Koornneef L, Berghout A, van der Gaag R. Effect of abnormal thyroid function on the severity of Graves' ophthalmopathy. Arch Intern Med. 1990;150:1098-1101.

34. Bertalena L, Marcocci C, Bogazzi F, et al. Relation between therapy for hyperthyroidism and the course of Grave's ophthalmopathy. $N$ Engl J Med. 1998;338:73-78.

35. Tallstedt L, Lundell G, Blomgren H, Bring J. Does early administration of thyroxine reduce the development of Graves' ophthalmopathy after radioiodine therapy? Eur J Endocrinol. 1994;130:494-497.

36. Prummel MR, Wiersinga WM. Smoking and risk of Graves disease. JAMA. 1993;269:479-482.

37. Nunery WR, Martin RT, Heinz GW, Gavin TJ. The association of cigarette smoking with clinical subtypes of ophthalmic Graves' disease. Ophthal Plast Reconstr Surg. 1993;9:77-82.

38. Pfeilschifter J, Ziegler R. Smoking and endocrine ophthalmopathy: impact of smoking severity and current vs lifetime cigarette consumption. Clin Endocrinol (Oxf). 1996;45:477-481.

39. Burch HB, Lahiri S, Bahn RS, Barnes S. Superoxide radical production stimulates retroocular fibroblast proliferation in Graves ophthalmopathy. Exp Eye Res. 1997;65:311-316.

40. Marcocci C, Bartalena L, Panicucci M, et al. Orbital cobalt irradiation combined with retrobulbar or systemic corticosteroids for Graves' ophthalmopathy: a comparative study. Clin Endocrinol (Oxf). 1987;27:33-42.

41. Marcocci C, Bartalena L, Tanda ML, et al. Comparison of the effectiveness and tolerability of intravenous or oral glucocorticoids associated with orbital radiotherapy in the management of severe Graves' ophthalmopathy: results of a prospective, single-blind, randomized study. J Clin Endocrinol Metab. 2001;86: 3562-3567.

42. Marino M, Lisi S, Pinchera A, et al. Glycosoaminoglycans provide a binding site for thyroglobulin in orbital tissues of patients with thyroidassociated ophthalmopathy. Thyroid. 2003;13:851-859.

43. Kahaly GJ, Pitz S, Hommel G, Dittmar M. Randomized, single-blind trial of intravenous versus oral steroid monotherapy in Graves' orbitopathy. J Clin Endocrinol Metab. 2005;90:5234-5240.

44. Bahn RS. The EUGOGO Consensus Statement of the Management of Graves' Orbitopathy: Equally Applicable to North American Clinicians and Patients. Thyroid. 2008;18:3:281-282. 
45. Juler FA. III. Diseases of the orbit. Acute purulent keratitis in exophthalmic goitre treated by repeated tarsorrhaphy, resection of cervical sympathetic and x-rays; retention of vision in one eye. Trans Ophthalmol Soc UK. 1913;33; 58-62.

46. Bartalena L, Marcocci C, Tanda ML, et al. Orbital radiotherapy for Graves' ophthalmopathy. Thyroid. 2002;12:245-250.

47. Wiersinga WM. Retrobulbar irradiation in Graves orbitopathy. The Dutch experience. Ophthal Plast Reconstr Surg. 2002;18:175-176.

48. Gorman CA, Garrity JA, Fatourechi V, et al. A prospective, randomized, double-blind, placebo-controlled study of orbital radiotherapy for Graves' ophthalmopathy. Ophthalmology. 2001;108:1523-1534.

49. Bradley EA, Gower EW, Bradley DJ, et al. Orbital radiation for Graves ophthalmopathy - A report by the American Academy of Ophthalmology. Ophthalmology. 2008;115:398-409.

50. Mourits MP, van Kempen-Harteveld ML, Garcia MB, Koppeschaar HP, Tick L, Terwee CB. Radiotherapy for Graves' orbitopathy: randomized placebo-controlled study. Lancet. 2000;355:505-509.

51. Prummel MF, Terwee CB, Gerding MN, et al. A randomized controlled trial of orbital radiotherapy versus sham irradiation in patients with mild Graves' ophthalmopathy. J Clin Endocrinol Metab. 2004;89:15-20.

52. Kuriyan AE, Phipps RP, Feldon SE. The eye and thyroid disease. Curr Opin Ophthalmol. 2008;19:499-506.

53. Banga JP, Nielsen CH, Gilbert JA, El Fassi D, Hegedus L. Application of New Therapies in Graves' Disease and Thyroid-Associated Ophthalmopathy: Animal Models and Translation to Human Clinic Trials. Thyroid. 2008;18(9):973-981.
54. Garrity JA, Bahn RS. Pathogenesis of Graves Ophthalmopathy: Implications for Prediction, Prevention, and Treatment. Am J Ophthalmol. 2006;142(1):147-153.

55. Dorner T. Crossroads of B cell activation in autoimmunity: rationale of targeting B cells. J Rheumatol Suppl. 2006;77:3-11.

56. El Fassi D, Nielsen CH, Hasselbalch HC, Hegedus L. Treatmentresistent sever, active Graves' ophthalmopathy successfully treated with B lymphocyte depletion. Thyroid. 2006;16:709-710.

57. Salvi M, Vannucchi G, Campi I, et al. Treatment of Graves' disease and associated ophthalmopathy with the anti-CD20 monoclonal antibody rituximab: an open study. Eur J Endocrinol. 2007;156:33-40.

58. Schneider H, Downey J, Smith A, et al. Reversal of the TCR stop signal by CTLA-4. Science. 2006;313:1972-1974.

59. Genvese MC, Becker JC, Schiff M, et al. Abatacept for rheumatoid arthritis refractory to tumor necrosis factor alpha inhibition. $N$ Engl $J$ Med. 2005;353:1114-1123.

60. Paridaens D, van den Bosch WA, van der Loos TL, Krenning EP, van Hagen PM. The effect of etanercept on Graves' ophthalmopathy: a pilot study. Eye. 2005;19:1286-1289.

61. Durrani OM, Reuser TQ, Murray PI. Infliximab: a novel treatment for sight-threatening thyroid associated ophthalmopathy. Orbit. $2005 ; 24: 117-119$
International Journal of General Medicine

\section{Publish your work in this journal}

The International Journal of General Medicine is an international, peer-reviewed open-access journal that focuses on general and internal medicine, pathogenesis, epidemiology, diagnosis, monitoring and treatment protocols. The journal is characterized by the rapid reporting of reviews, original research and clinical studies across all disease areas.

\section{Dovepress}

A key focus is the elucidation of disease processes and management protocols resulting in improved outcomes for the patient.The manuscript management system is completely online and includes a very quick and fair peer-review system. Visit http://www.dovepress.com/ testimonials.php to read real quotes from published authors. 